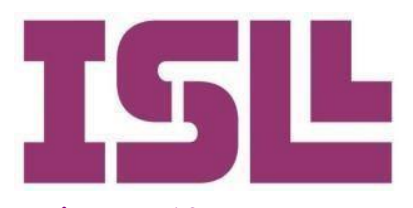

Número 10

Diciembre de 2018

Pág. 95 a la 117

\section{Palabras clave}

Lectura, prueba de lectura, estudiante universitario

\title{
Diseño y validación de una prueba piloto para la evaluación de la competencia lectora de estudiantes universitarios
}

\author{
Andrea Felipe Morales \\ Universidad de Málaga \\ Juan de Dios Villanueva Roa \\ Universidad de Granada
}

Recibido: 01-07-2018

Aceptado: 30-09-2018

\section{Resumen:}

En este artículo se presenta una prueba piloto diseñada con el objetivo de evaluar la competencia lectora de estudiantes universitarios, para quienes no existe hasta el momento ninguna prueba validada en español. Tras una breve aproximación al marco teórico que sustenta la prueba -denominada Test de Competencia Lectora para Universitarios (Test CLUni) (Felipe, Barrios, Villanueva y Caldevilla)-, se procede a la descripción del test, que se compone de 12 textos -9 de ellos auténticos, y 3 elaborados ad hoc- seleccionados considerando la tipología textual que propone el informe PISA-2015: 2 instructivos, 2

narrativos, 2 expositivos, 2 argumentativos, 2 descriptivos y 2 transaccionales, de los que 6 son continuos y 6 discontinuos. Los 36 ítems que conforman el test están elaborados asimismo teniendo en cuenta el marco teórico de PISA en cuanto a inclusión de las tres categorías de aspectos que definen el marco de lectura en PISA: acceder y recuperar, integrar e interpretar, y reflexionar y evaluar. A continuación, se expone el proceso de validación al que se ha sometido la prueba, que incluyó validación del contenido mediante juicio de expertos de las áreas de Didáctica de la Lengua y la Literatura, Filología Hispánica, Lingüística y Comunicación. Se presentan asimismo los resultados que se obtuvieron en esta fase de pilotaje en una muestra de 65 estudiantes de los Grados en Educación Infantil, Educación Primaria y Comunicación Audiovisual de la Universidad de Málaga- y en las valoraciones de los expertos, y se analiza la percepción del alumnado participante sobre algunos aspectos relacionados con la prueba ( $\mathrm{p}$. ej., la dificultad percibida). El artículo concluye exponiendo los cambios que se plantean introducir en el test derivados del proceso de validación. 


\section{Introducción}

Como describe Solé (1992), leer es un proceso de interacción entre el lector y el texto mediante el cual el lector trata de satisfacer de una forma activa, procesando y examinando el texto, los diversos objetivos que motivan su lectura, siendo estos imprescindibles para que tenga lugar dicho proceso. El lector construye el significado del texto según sus experiencias y conocimientos previos y guiado por sus objetivos. Los objetivos del lector condicionan, por tanto, su lectura, a pesar de que el texto sea invariable, y son esenciales porque no solo determinan las estrategias de lectura de las que depende la comprensión, sino que influyen en el control inconsciente que tenemos sobre este proceso.

Según la Organización para la Cooperación y el Desarrollo Económicos (OECD) la competencia lectora consiste en "la capacidad de un individuo para comprender, utilizar, reflexionar y comprometerse con textos escritos para alcanzar sus objetivos, desarrollar sus conocimientos y potencial, y participar en la sociedad" (Ministerio de Educación, 2010, p. 34). Es la práctica de la lectura en sus diversos formatos, respondiendo a las necesidades que se presenten a lo largo de la vida, la que puede favorecer que el lector alcance una competencia lectora conforme a los objetivos de cada situación, y esta debe ser desarrollada y evaluada durante todo el proceso educativo del alumnado y no tan solo en las etapas iniciales. Como señala Solé (2012),

Confinar el aprendizaje de esta competencia a la adquisición inicial de los debutantes es extremadamente restrictivo: es necesario aprender a leer, por supuesto. Pero también es necesarioaprender a leer para aprender, para pensar, para disfrutar. En la época de la sobreinformación, saber leer con criterio, de forma inteligente y reflexiva es tal vez un bien más preciado que nunca. Formar lectores equivale a formar ciudadanos que puedan elegir la lectura para una variedad de propósitos, que sepan qué leer y cómo hacerlo, que puedan utilizarla para transformar la información en conocimiento. (p. 50).

Diversos estudios evidencian que los estudiantes universitarios tienen dificultades en la interpretación de textos, en encontrar las ideas principales o en la generación de una opinión crítica con respecto al texto (Arnoux, 2007; Calderón \& Quijano, 2010; De Brito \& Angeli, 2005; Felipe \& Barrios, 2015; Irigoren, Jiménez \& Acuña, 2008; Martínez Solís, 1997; Velásquez, Cornejo, Valderrama \& Roco, 2008). Para Delgadová y Gullerová (2015):

la competencia lectora es un complejo de habilidades y capacidades lectoras necesarias para el trabajo efectivo con el texto. No es solo la habilidad de leer palabras, frases y textos; significa también comprender todo lo leído, trabajar con el contenido y con la información obtenida para interpretarla y transmitirla correctamente y, sobre todo, para crear nuestras propias ideas a partir de la información obtenida, es decir, crear un nuevo conocimiento innovador a partir del conocimiento adquirido a través de la lectura. (p. 49). 
Por otro lado, la lectura que realizan los estudiantes actualmente se encuentra en las redes, en Internet. La mayoría de los jóvenes estudiantes creen encontrar en las redes las respuestas que buscan, si bien no estamos convencidos de que sean las que necesitan. Estamos de acuerdo con Solé (2012) cuando dice que:

esta lectura posmoderna o hermenéutica hace más perentoria aún la necesidad de contribuir a formar lectores activos, dotados de criterio, capaces de combinar la lectura rápida y muchas veces superficial que a menudo requiere la red con la capacidad de concentrarse en la lectura lineal de textos narrativos o expositivos. (p. 48)

Como indican Martínez, Vidal-Abarca, Sellés y Gilabert (2008), la carencia de un modelo teórico que adolece la mayoría de los tests de comprensión en español "les lleva a referirse a capacidades genéricas de comprensión sin una articulación suficiente entre las capacidades y los ítems que supuestamente miden las primeras" (p. 321). En el Test de Competencia Lectora para Universitarios se ha procurado que el lector se enfrente a textos auténticos que recogen las diversas ópticas y enfoques de las situaciones que puede encontrarse en la vida cotidiana, con el fin de que las respuestas reflejen la realidad más próxima a su comprensión lectora, a la aplicación de los contenidos en el día a día en el que se mueven los lectores, y en analizar su espíritu crítico con lo que acaban de leer, tratando de resolver la problemática de adecuación y eficiencia que se ha presentado con anterioridad en otros estudios.

\section{Objetivos}

En el trabajo que presentamos a continuación pretendemos mostrar el proceso de diseño y validación de una prueba de competencia lectora - el Test de Competencia Lectora para Universitarios: Test CLUni (Felipe, 2014; Felipe \& Barrios, 2015, 2017)mediante la que se trata de analizar el nivel de esta competencia en estudiantes universitarios. Asimismo, se pretende evaluar la prueba a partir de los datos de validación obtenidos con el fin de introducir mejoras a la misma.

\section{Método}

\section{Participantes}

Participaron en el estudio un total de 65 estudiantes universitarios, de los que 45 son mujeres $(69,2 \%)$ y 20 hombres $(30,8 \%)$, de edades comprendidas entre los 20 y los 55 años (media de edad: 23 años y 8 meses; D.T.: 5 años y un mes; moda: 21). Se trataba de alumnado de cuarto curso de los Grados en Comunicación Audiovisual $(n=15)$, Educación Primaria $(n=32)$ y Educación Infantil $(n=18)$ de la Universidad de Málaga en el curso académico 2017-2018. 


\section{Instrumento}

El Test CLUni se aplicó en horario lectivo, cedido por los docentes a cargo de los grupos. Los participantes disponían de un máximo de 1 hora y media para completar la prueba. Se les solicitó que, tras cumplimentar una serie de datos académicos y biográficos, respondiesen a las preguntas marcando o escribiendo en un cuadernillo de preguntas, utilizando para ello los textos correspondientes a cada una de ellas -ubicados en el cuadernillo de textos-, pudiendo releerlos y consultarlos tantas veces como fuese necesario, dentro del límite temporal establecido.

La prueba consta de un total de 12 textos seleccionados considerando la tipología textual que propone el informe PISA-2015 (OECD, 2017) -según una tipología adaptada de Werlich (1976) utilizada en PISA-2009/2015 (OECD, 2009, 2017)- (Tabla 1). Los 36 ítems que componen de la prueba -17 abiertos y 19 cerrados- fueron diseñados asimismo teniendo en cuenta el marco teórico de PISA-2015, con el propósito de evaluar los tres aspectos de la competencia lectora que establece dicho informe. Un total de 5 preguntas evalúan la categoría del aspecto de acceso y obtención de la información, 20, la de la integración e interpretación y 11, la de reflexión y valoración. En el proceso de selección de los textos y de elaboración de los ítems se siguieron las pautas que ofrecieron Mendelovits y Sainsbury en su conferencia 'Cómo construir ítems de Lengua' (2013).

Tabla 1. Clasificación de los textos que componen el Test CLUni

\begin{tabular}{|c|c|c|c|}
\hline$N^{o}$ & Tipo de texto & Formato & $\begin{array}{c}\text { Situaciones/ } \\
\text { contextos de } \\
\text { lectura }\end{array}$ \\
\hline 1 & Instructivo (modo de actuación) & Continuo & Educativo \\
\hline 2 & Narrativo (novela) & Continuo & Personal \\
\hline 3 & Instructivo (normas) & $\begin{array}{c}\text { Discontinu } \\
\text { o }\end{array}$ & Educativo \\
\hline 4 & Expositivo (representaciones gráficas) & $\begin{array}{l}\text { Diseontint } \\
\qquad 0\end{array}$ & Público \\
\hline 5 & $\begin{array}{l}\text { Argumentativo (artículo de opinión en un } \\
\text { blog) }\end{array}$ & $\begin{array}{r}\text { Continuo } \\
\text { Discontinu } \\
\end{array}$ & Público \\
\hline & & 0 & Público \\
\hline 7 & Descriptivo (plano) & $\begin{array}{c}\text { Discontinu } \\
\text { o }\end{array}$ & Público \\
\hline
\end{tabular}




\begin{tabular}{cccc}
\hline $\mathbf{8}$ & Descriptivo (guía) & Continuo & Público \\
\hline $\mathbf{9}$ & Expositivo (ensayo académico) & Continuo & Educativo \\
\hline $\mathbf{1 0}$ & Narrativo (relato) & Continuo & Personal \\
\hline $\mathbf{1 1}$ & Transaccional (anuncio de alquiler) & Discontinu & Personal \\
& & 0 & \\
\hline $\mathbf{1 2}$ & Transaccional (chats derivados del texto 11) & Discontinu & Personal \\
& & 0 & \\
\hline
\end{tabular}

La prueba consta de un total de 12 textos seleccionados considerando la tipología textual que propone el informe PISA-2015 (OECD, 2017) -según una tipología adaptada de Werlich (1976) utilizada en PISA-2009/2015 (OECD, 2009, 2017)- (Tabla 1). Los 36 ítems que componen de la prueba -17 abiertos y 19 cerrados- fueron diseñados asimismo teniendo en cuenta el marco teórico de PISA-2015, con el propósito de evaluar los tres aspectos de la competencia lectora que establece dicho informe. Un total de 5 preguntas evalúan la categoría del aspecto de acceso y obtención de la información, 20, la de la integración e interpretación y 11, la de reflexión y valoración. En el proceso de selección de los textos y de elaboración de los ítems se siguieron las pautas que ofrecieron Mendelovits y Sainsbury en su conferencia 'Cómo construir ítems de Lengua' (2013).

\section{Procedimiento de análisis de los datos}

En este estudio se ha realizado un análisis de consistencia interna del Test CLUni basado en el coeficiente alfa de Cronbach. Asimismo, con respecto a cada ítem se han calculado los índices de dificultad y discriminación. Finalmente, se realizó un análisis de la validez de la prueba, así como un análisis estadístico de los datos contenidos con el instrumento de autoinforme de valoración de la misma por parte de los participantes.

\section{Resultados}

\section{Consistencia interna}

El coeficiente alfa de Cronbach de la puntuación global obtenida por los participantes fue 805, lo cual supone un índice de consistencia aceptable (Nunnally, 1978) y mucho más alto que el mínimo de ,550 que se establece para constructos moderadamente amplios (Van DeVen \& Ferry, 1980) como es el caso del de la competencia lectora. 


\section{Análisis de los ítems}

Los datos más relevantes obtenidos a partir del análisis de los ítems del Test CLUni se muestran en la tabla 2. En la segunda columna figuran los índices de dificultad de los ítems, que presentan valores entre, 185 y ,908. Según la clasificación que propone Gil (2011, p. 58), la mayoría de los ítems de la prueba $(n=24)$ quedarían incluidos en las categorías de "muy fáciles" y "fáciles" - con índices de dificultad superiores a ,55-. El resto de los ítems podrían considerarse "normales" $(n=2)$, "difíciles" ( $n=7)$ y "muy difíciles" ( $n=3)$. Es presuponible que el índice de dificultad de los ítems disminuya aumentando así la dificultad de estos- al aplicar el test a alumnado de cursos inferiores, ya que, como se evidenció en un estudio previo (Felipe, 2015), el rendimiento del alumnado de $4^{\circ}$ curso en una versión anterior del Test CLUni es superior al de $1^{\circ}$. En la cuarta columna aparecen los índices de discriminación de los ítems. Siguiendo, de nuevo, la clasificación que propone Gil (2011, p. 61), 16 de los ítems discriminan muy bien $(n=4)$ o bien $(n=12), 12$ de los ítems discriminan poco y 8 de los ítems se deben mejorar $(n=6)$ o carecen de utilidad para discriminar $(n=2)$. La última columna muestra los valores del alfa total de la prueba asociados a la supresión de cada ítem. Estos valores demuestran que la eliminación de los algunos ítems supondría un incremento mínimo del índice de fiabilidad.

Tabla 2. Estadísticos obtenidos en el análisis de los ítems del Test CLUni

\begin{tabular}{|c|c|c|c|c|}
\hline $\begin{array}{c}\text { Ítem } \\
s\end{array}$ & $\begin{array}{c}\text { Índice } \\
\text { de dificultad }\end{array}$ & D.T. & $\begin{array}{c}\text { Índice } \\
\text { de } \\
\text { discriminación }\end{array}$ & $\begin{array}{c}\text { Alfa de } \\
\text { Cronbach } \\
\text { si el elemento } \\
\text { se ha suprimido }\end{array}$ \\
\hline 1 & ,323 & ,4713 & ,461 & ,794 \\
\hline 2 & ,462 &, 5024 & ,266 &, 802 \\
\hline 3 & ,908 & ,2917 & ,019 & ,808 \\
\hline 4 & ,615 & ,4903 & ,238 & ,803 \\
\hline 5 & ,708 & ,4584 & ,315 & ,800 \\
\hline 6 & ,754 & ,4341 & ,299 & ,801 \\
\hline 7 & ,323 & ,4713 & ,257 & ,802 \\
\hline 8 & 692 & ,4651 & ,394 & ,797 \\
\hline 9 & ,615 & ,4903 & ,176 & ,806 \\
\hline
\end{tabular}




\begin{tabular}{|c|c|c|c|c|}
\hline 10 & ,246 & ,4341 & ,343 & ,799 \\
\hline 11 & ,646 & ,4819 & , 100 & ,809 \\
\hline 12 & ,346 & ,3298 & ,338 & ,800 \\
\hline 13 & ,908 & ,2917 & ,215 & ,803 \\
\hline 14 & ,415 & ,4966 & ,067 & ,810 \\
\hline 15 & ,769 & ,4246 & ,350 & ,799 \\
\hline 16 & ,831 & 3779 & ,275 & ,801 \\
\hline 17 & ,662 & ,4769 & ,601 & ,788 \\
\hline 18 & ,969 & , 1740 & , 165 &, 805 \\
\hline 19 & ,892 & ,3124 & ,312 & ,801 \\
\hline 20 & , 185 & ,3910 & ,347 & ,799 \\
\hline 21 & ,354 & ,4819 & ,344 & ,799 \\
\hline 22 & ,677 & ,4713 & ,379 & ,797 \\
\hline 23 & , 185 & ,3910 & ,254 & ,802 \\
\hline 24 & ,446 & ,5010 & ,344 & ,799 \\
\hline 25 & ,831 & ,3779 & ,223 & ,803 \\
\hline 26 & ,508 & ,5038 & ,276 & ,802 \\
\hline 27 & ,677 & ,4713 & ,271 & ,802 \\
\hline 28 & ,846 & ,2784 & ,239 & ,803 \\
\hline 29 & ,662 & ,4769 & ,220 & ,804 \\
\hline 30 & ,862 & ,3481 & ,153 & ,805 \\
\hline 31 & ,969 & ,1740 & ,334 & ,802 \\
\hline 32 & ,877 & ,3311 & ,173 & 804 \\
\hline 33 & ,985 & ,1240 & ,103 & ,806 \\
\hline 34 & ,415 & ,4966 & ,350 & ,799 \\
\hline 35 & ,554 & ,5010 & ,632 & ,786 \\
\hline 36 & ,769 & ,4246 & ,496 & ,793 \\
\hline
\end{tabular}


El Test CLUni fue validado por 6 expertos de las áreas de Didáctica de la Lengua y la Literatura, Filología Hispánica, Lingüística y Comunicación), a quienes se les envió por correo electrónico la versión preliminar de la prueba (cuadernillo de textos, cuadernillo de preguntas y clave de respuestas) y un documento -elaborado siguiendo las pautas y consejos de Feliz (2010) - en el que se les solicitaba su colaboración para su validación por expertos. Tras una breve introducción en la que se describía la prueba y sus destinatarios, se les presentaba una serie de tablas para una valoración general de la prueba y para la valoración específica de cada uno de los textos y los ítems que se refieren a ellos, en cuanto a su pertinencia (en qué modo evalúa la competencia lectora) y adecuación (a los destinatarios de la prueba), mediante escalas de 1 a 5 , en las que 1 significaba "nada pertinente/adecuado" y 5, "muy pertinente/adecuado". Disponían, además, de un espacio para comentarios sobre la prueba en general y sobre cada texto e ítem, donde podían anotar lo que considerasen oportuno (motivos de su elección en la escala, propuestas de mejora, propuestas de cambio o eliminación, erratas...). Por último se les invitaba a escribir comentarios y sugerencias.

Los expertos que participaron en el proceso de validación valoraron muy positivamente la mayoría de los textos e ítems que componen la prueba. Asimismo, en sus comentarios generales señalaron que consideraban que el Test CLUni es unaherramienta pertinente y válida, que destaca por la variedad textual, y que tiene validez de constructo ya que sus ítems están en correspondencia con los indicios que se derivan del concepto y la capacidad que se pretende medir. Consideraron, finalmente, que la prueba se ajusta a los objetivos pretendidos y que el instrumento está preparado (a excepción de leves consideraciones) para su aplicación y recogida de datos.

No obstante, los expertos consultados señalaron determinados aspectos de mejora, que fueron considerados y se buscó el consenso para realizar las modificaciones pertinentes, como, por ejemplo, la reducción del tiempo para su realización (que inicialmente era de una hora y media), debido a que la amplitud de la prueba y la longitud de algunos textos puede suponer fatiga y una demanda cognitiva excesiva.

Asimismo, la validez del contenido se procuró mediante la selección de tipos de textos y el diseño de los ítems conforme a los supuestos establecidos en el marco teórico de PISA 2009-2015.

\section{Baremación}

Se realizó la baremación de la prueba según las puntuaciones totales obtenidas por los participantes en el estudio (Tabla 3). Como podemos observar, la media de las puntuaciones fue de 22,89 sobre 36 , que equivaldría a un 6,36 sobre 10 . Los estudiantes 
que obtuvieron mejores puntuaciones en la prueba contestaron correctamente 31 de las preguntas (lo que supondría una calificación de 8,61 sobre 10) y los alumnos con una puntuación más baja respondieron de forma acertada tan solo 10 ítems (lo que correspondería a una nota de 2,78 sobre 10 ).

Tabla 3. Estadísticos de la puntuación total de los participantes en la prueba

\begin{tabular}{lccccc}
\hline Media & D.T. & Moda & $\begin{array}{c}\text { Median } \\
a\end{array}$ & Mín. & \\
& & & & & \\
22,89 & 5,392 & 28,5 & 23 & 10 & 31 \\
\hline
\end{tabular}

\section{Valoración de los participantes}

A los estudiantes se les distribuyó un cuestionario de valoración de aspectos de la prueba $C L U n i$ y de reacción a la misma al finalizarla, cuyos hallazgos se presentan en esta sección. Respecto de la extensión de la prueba, el 29\% de los participantes opinaron que era demasiado larga; asimismo, el 21,9\% manifestó que su extensión había afectado a su rendimiento; quienes expusieron de qué manera la longitud de la prueba les afectó en la realización de la misma se referían, en la mayoría de los casos, a que a medida de que transcurría el tiempo disminuía su concentración y atención, y aumentaba su cansancio. A este respecto cabe señalar que el tiempo medio de realización fue de 53 minutos). Estos datos -al igual que los procedentes de los comentarios de los expertos consultadosaconsejan revisar la misma con el objetivo de reducir el tiempo de realización. En otro orden de cosas, más de la mitad de los participantes $(59,4 \%)$ afirmó que el test le había supuesto un reto y, a excepción de uno de ellos, el resto consideró interesante realizar este tipo de pruebas. También el conjunto de los participantes - excepto uno- manifestó su interés por conocer el resultado de la prueba.

Asimismo, se les solicitó que valorasen la dificultad de la prueba en una escalade 1 (= Ninguna dificultad) a 10 (= Mucha dificultad). De los datos obtenidos se desprende que el grupo de participantes consideró que la prueba planteaba una dificultad media aunque, a tenor de los valores de la moda y la mediana, un número considerable de ellos manifestó que la prueba entrañaba una dificultad alta (Tabla 4). 
Tabla 4. Estadísticos de la dificultad del TestCLUni percibida

\begin{tabular}{cccccc}
\hline Media & D.T. & Moda & Mediana & Mín. & \\
6,36 & 2,028 & 8 & 7 & 1 & 8 \\
\hline
\end{tabular}

Finalmente se les solicitó que estimaran el número de preguntas que creían haber respondido correctamente; como puede apreciarse en los resultados obtenidos (Tabla 5), los participantes, como conjunto, sobreestiman su rendimiento en esta prueba, lo que puede llevar a pensar en la posibilidad de que sobreestimen asimismo su nivel de competencia lectora.

Tabla 5. Autopercepción de los resultados en el TestCLUni

\begin{tabular}{cccccc}
\hline Media & D.T. & Moda & $\begin{array}{c}\text { Median } \\
\boldsymbol{a}\end{array}$ & Mín. & Máx. \\
& & & & & \\
28,21 & 5,366 & 30 & 30 & 15 & \\
\hline
\end{tabular}

\section{Conclusiones}

Este artículo pretende presentar el proceso de diseño y validación de una prueba de competencia lectora en fase piloto orientada a evaluar la competencia lectora de estudiantes universitarios. A la luz de los resultados obtenidos, en la siguiente fase de su desarrollo se deberán introducir ciertos cambios en el diseño de la misma, destinados a eliminar ítems con un índice bajo de discriminación y a reducir su extensión para reducir el riesgo de que la fatiga incida en el rendimiento en la prueba. Asimismo, somos conscientes de que es necesario ampliar el número de participantes -así como su procedencia tanto geográfica como de estudios universitarios- en la investigación que evalúe la próxima prueba piloto con el fin de incrementar la representatividad de la muestra. No obstante, entendemos que el Test CLUni es una aportación al ámbito de la evaluación de la competencia lectora para una población para la que, hasta la fecha, escasean instrumentos validados. 


\section{Referencias}

Arnoux, E. N., Nogueira, S. y Silvestri, A. (2007). Habilidades metacomprensivas en estudiantes de profesorado: la formulación de preguntas. Folios: Revista de la Facultad de Humanidades, (25), 81-95.

Calderón, A., y Quijano, J. (2010). Características de comprensión lectora en estudiantes universitarios. Estudios Socio-Jurídicos, 12(1), 337-364. Recuperado de: http://revistas.urosario.edu.co/index.php/sociojuridicos/article/view/1195

De Brito, N. y Angeli, A. (2005). Comprensión lectora en universitarios cursantes del primer año en distintas carreras. Paradigma, 26(2), 99-113.

Delgadová, E. y Gullerová, M. (2015). Comprensión lectora. Un estudio sobre la competencia lectora en el contexto universitario. Lenguajes y Textos, 41, mayo, 4553.

Felipe, A. (2014). Elaboración de una prueba piloto para la competencia lectora de estudiantes universitarios. En Requeijo, P. y Gaona, C. (coord.). Contenidos innovadores en la Universidad actual (pp. 297-311). Madrid: McGraw-Hill.

Felipe, A. y Barrios, E. (2015). Prospective teachers' reading competence: percepcions and performance in a reading test. Procedia - Social and Behavioral Sciences, 178, 87-93. Recuperado de: https://www.sciencedirect.com/science/article/pii/S187704281501944

Felipe, A. y Barrios, E. (2017). Evaluación de la competencia lectora de futuros docentes, Investigaciones Sobre Lectura, 7, 7-21.

Feliz, T. (2010). Validación de un cuestionario por expertos. Recuperado de: https://www.youtube.com/watch?v=C7S5ImJZhvo.

Gil, J.A. (2011). Técnicas e instrumentos para la recogida de información. Madrid: Universidad Nacional de Educación a Distancia.

Irigoyen, J., Jiménez, M., y Acuña, K. (2008). Análisis de la competencia lectora en estudiantes universitarios. Boletín electrónico de investigación de la Asociación Oaxaqueña de Psicología, 4, 84-96. Recuperado de: http://www.conductitlan.net/52_competencia_lectora.pdf

Martínez, T.; Vidal-Abarca, E.; Sellés, P. y Gilabert, R. (2008). Evaluación de las estrategias y procesos de comprensión: el Test de Procesos de Comprensión. Infancia y Aprendizaje, 31(3), 319-333. 
Martínez Solís, M. C. (1997). El desarrollo de estrategias discursivas a nivel universitario. En M.C. Martínez (Comp.), Los procesos de la lectura y la escritura (11-41). Santiago de Cali: Universidad del Valle.

Ministerio de Educación (2010). La lectura en PISA 2009. Marcos y pruebas de evaluación. Madrid. Recuperado http://www.mecd.gob.es/dctm/ievaluacion/internacional/lectura-enpisa.pdf?documentId $=0901 \mathrm{e} 72 \mathrm{~b} 8072 \mathrm{f} 8 \mathrm{~d} 9$

Mendelovits, J. y Sainsbury, M. (2013). 'Cómo construir ítems de Lengua', en el seminario Presentación de ítems liberados de PISA, TIMSS y PIRLS. Marco teórico, elaboración y aprovechamiento didáctico, Centro Internacional de Estudios Superiores de Español, Fundación Comillas, Comillas (Cantabria). Disponible en: https://www.youtube.com/watch? $\mathrm{v}=\mathrm{cN} 8 \mathrm{Kgl}-\mathrm{klOY} \quad$ (primera parte) y https://www.youtube.com/watch?v=SN6CXVf_Ei8 (segunda parte).

Nunnally, J. C. (1978). Psychometric theory (2nd ed.). Nueva York: McGraw-Hill.

OECD (2009). PISA 2009 Assessment Framework - Key competencies in reading, Mathematics and Science. París: PISA, OECD Publishing. Recuperado de: www.oecd.org/dataoecd/11/40/44455820.pdf

OECD (2017). PISA 2015 Assessment and Analytical Framework: Science, Reading, Mathematic, Financial Literacy and Collaborative Problem Solving, revised edition, París: PISA, OECD Publishing. Recuperado de http://dx.doi.org/10.1787/9789264281820-en.

Solé, I. (1992). Estrategias de lectura. Barcelona, España: Graó.

Solé, I. (2012). Competencia lectora y aprendizaje. Revista Iberoamericana de Educación (OEI), 2012, 59, 43-61.

Van Deven A. H. y Ferry, D. L. (1980). Measuring and Assessing Organizations. Nueva York: John Wiley y Sons, Inc.

Velásquez Rivera, M., Cornejo, C., y Roco, Á. (2008). Evaluación de la competencia lectora en estudiantes de primer año de carreras del área humanista y carreras del área de la salud en tres universidades del Consejo de Rectores. Estudios pedagógicos (Valdivia), 34(1), 123-138.

Weirlich, E. (1976). A text grammar of English. Heidelberg: Quelle and Meyer. 


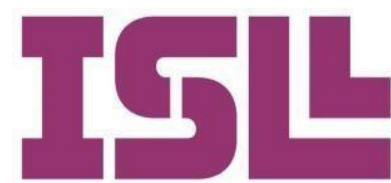

Number 10

2018 December

\title{
Design and validation of a pilot test to assess university students' reading competence
}

\author{
Andrea Felipe Morales \\ Universidad de Málaga \\ Juan de Dios Villanueva Roa \\ Universidad de Granada
}

Received: 01-07-2018

Accepted: $30-09-2018$

Pag. 95 to 117

\section{Keywords}

Reading, reading test university students

\begin{abstract}
:
\end{abstract}
This paper presents a pilot test designed to assess university students' reading competence. As yet there is no validated test of reading competence in Spanish for this population. After a brief introduction to the theoretical framework underlying this test - Reading Competence Test for University Students (Felipe, Barrios, Villanueva y Caldevilla)-, the test is des-cribed. It consists of 12 texts: 9 of them authentic texts and the remaining three were written for the purpose of the test. These texts were selected according to the text types in PISA2015: 2 instructive, 2

narrative, 2 expository, 2 argumentative, 2 descriptive y 2 transactional texts; additionally, 6 are continuous and 6, dis-continuous texts. The 36 items that comprise the test were also developed accord-ing to the PISA-2015 reading framework with the aim to include the three aspect categories that define the PISA reading literacy framework (access and retrieve, integrate and interpret, and reflect and evaluate). The test validation process is then described; it consisted on the evaluation of the content validity by a group of experts from the areas of Language and Literature Didactics, Hispanic Philology, Linguistics and Communication. Results obtained from 65 students from the Degrees of Preprimary Education, Primary Education and Audio-visual Communication are also presented, alongside the experts' evaluation and comments, and the test takers' perceptions of issues concerning the test (e.g., perceived difficult-y). The paper concludes with the proposed changes to the test in light of the process of validation. 


\section{Introduction}

As Solé (1992) describes, reading is a process of interaction between the reader and the text through which the reader tries to satisfy in an active way, processing and examining the text, the various objectives that motivate their reading, being essential for process to take place. The reader constructs the meaning of the text according to their previous experiences and knowledge and guided by their objectives. The objectives of the reader therefore condition their reading, despite the fact that the text is invariable, and are essential because they not only determine the reading strategies on which understanding depends, but influence the unconscious control we have on this process.

According to the Organization for Economic Co-operation and Development (OECD), reading competence consists of "the ability of an individual to understand, use, reflect and engage with written texts, in order to achieve one's goals, to develop one's knowledge and potential, and participate in society"(Ministerio de Educación, 2010, p.34). It is the practice of reading in its various formats, responding to the needs that arise throughout life, which can help the reader reach a reading competence according to the goals of each situation, and this must be developed and evaluated throughout the educational process of the students and not only in the initial stages. As Solé (2012) points out,

Confinar el aprendizaje de esta competencia a la adquisición inicial de los debutantes es extremadamente restrictivo: es necesario aprender a leer, por supuesto. Pero también es necesario aprender a leer para aprender, para pensar, para disfrutar. En la época de la sobre información, saber leer con criterio, de forma inteligente y reflexiva es tal vez un bien más preciado que nunca. Formar lectores equivale a formar ciudadanos que puedan elegir la lectura para una variedad de propósitos, que sepan qué leer y cómo hacerlo, que puedan utilizarla para transformar la información en conocimiento. (p. 50)

Several studies show that university students have difficulties in interpreting texts, in finding the main ideas or in generating a critical opinion about the text (Arnoux, 2007, Calderón \& Quijano, 2010, De Brito \& Angeli, 2005; Felipe \& Barrios, 2015; Irigoren, Jiménez \& Acuña, 2008; Martínez Solís, 1997; Velásquez, Cornejo, Valderrama \& Roco, 2008). For Delgadová and Gullerová (2015):

la competencia lectora es un complejo de habilidades y capacidades lectoras necesarias para el trabajo efectivo con el texto. No es solo la habilidad de leer palabras, frases y textos; significa también comprender todo lo leído, trabajar con el contenido y con la información obtenida para interpretarla y transmitirla correctamente y, sobre todo, para crear nuestras propias ideas a partir de la información obtenida, es decir, crear un nuevo conocimiento innovador a partir del conocimiento adquirido a través de la lectura. (p. 49) 
On the other hand, the reading that students are currently doing is in the networks, on the Internet. Most young students believe that they find the answers they are looking for in the networks, although we are not convinced that they are the ones they need. We agree with Solé (2012) when she says that:

esta lectura posmoderna o hermenéutica hace más perentoria aún la necesidad de contribuir a formar lectores activos, dotados de criterio, capaces de combinar la lectura rápida y muchas veces superficial que a menudo requiere la red con la capacidad de concentrarse en la lectura lineal de textos narrativos o expositivos. (p. 48)

As indicated by Martínez, Vidal-Abarca, Sellés and Gilabert (2008), most comprehension tests in Spanish suffer from the lack of a theoretical model "les lleva a referirse a capacidades genéricas de comprensión sin una articulación suficiente entre las capacidades y los ítems que supuestamente miden las primeras” (p. 321). In the Reading Competence Test for University students, the reader has been confronted with authentic texts that include the different perspectives and approaches to situations that can be found in everyday life, so that the answers reflect the reality closest to his reading comprehension, to the application of the contents in the day to day in which move the readers, and in analysing their critical spirit with what they have just read, trying to solve the problem of adequacy and efficiency that has been presented previously in other studies.

\section{Objectives}

In the work that we present below, we intend to show the design and validation process of a reading competence test -the Reading Competency Test for University students: CLUni Test (Felipe, 2014; Felipe \& Barrios, 2015, 2017)- by means of which tries to analyze the level of this competence in university students. It also aims to assess the evidence from the validation data obtained in order to make improvements to it.

\section{Method}

\section{Participants}

A total of 65 university students participated in the study, of which 45 are women (69.2\%) and 20 men (30.8\%), between the ages of 20 and 55 (average age: 23 years old and 8 months, DT: 5 years and a month, mode: 21). They were students in the fourth year of the Degrees in Audiovisual Communication $(n=15)$, Primary Education $(n=32)$ and Pre-Primary Education $(n=18)$ of the University of Málaga in the academic year 20172018. 


\section{Instrument}

The CLUni Test was applied during school hours, assigned by the teachers in charge of the groups. Participants had a maximum of 1 hour and a half to complete the test. They were asked to fill in a series of academic and biographical data, answering the questions by marking or writing in a question book, using the texts corresponding to each one of them -located in the textbook-, so that they can be read again and be consulted as many times necessary, within the established time limit.

The test consists of a total of 12 texts selected considering the textual typology proposed by the PISA-2015 report (OECD, 2017) -according to an adapted typology by Werlich (1976) used in PISA-2009/2015 (OECD, 2009, 2017)- (Table 1). The 36 items that make up the test -17 open and 19 closed- were designed like this taking into account the theoretical framework of PISA-2015, with the purpose of evaluating the three aspects of the reading competence established in said report. A total of 5 questions evaluate the category of access and retrieve aspect, 20, integrate and interpret, and 11, reflect and evaluate. In the process of selecting the texts and developing the items, the guidelines offered by Mendelovits and Sainsbury in their lecture 'How to construct Language items' (2013) were followed.

Table 1. Classification of texts used in CLUni Test

\begin{tabular}{cccc}
\hline $\mathrm{N}^{\mathbf{2}}$ & Type of text & Format & $\begin{array}{c}\text { Reading } \\
\text { situations }\end{array}$ \\
\hline 1 & Instructional (how to use) & Continuous & Educational \\
\hline 2 & Narrative (novel) & Continuous & Personal \\
\hline 3 & Instructional (rules) & Discontinuous & Educational \\
\hline 4 & Expository (graphic representation) & Discontinuous & Public \\
\hline 5 & Argumentative (op-ed blogs article) & Continuous & Public \\
\hline 6 & Argumentative (comments of text 5) & Discontinuous & Public \\
\hline 7 & Descriptive (plane) & Discontinuous & Public \\
\hline 8 & Descriptive (guide) & Continuous & Public \\
\hline 9 & Expository (academic essay) & Continuous & Educational \\
\hline 10 & Narrative (story) & Continuous & Personal \\
\hline 11 & Transactional (rental announcement) & Discontinuous & Personal \\
\hline 12 & Transactional (chats derived from text 11) & Discontinuous & Personal \\
\hline
\end{tabular}




\section{Data analysis}

In this study, an internal consistency analysis of the CLUni Test based on the Cronbach alpha coefficient was performed. Likewise, with respect to each item, the indices of difficulty and discrimination have been calculated. Finally, an analysis of the validity of the test was carried out, as well as a statistical analysis of the data contained with the self-report instrument for its evaluation by the participants.

\section{Results}

\section{Internal consistency}

The Cronbach's alpha coefficient of the overall score obtained by the participants was .805, which is an acceptable consistency index (Nunnally, 1978) and much higher than the minimum of .550, which is set for moderately broad constructs (Van DeVen \& Ferry, 1980) as is the case of reading comprehension.

\section{Analysis of the items}

The most relevant data obtained from the analysis of the items of the CLUni Test are shown in table 2. In the second column, the indices of difficulty of the items are shown, which have values between .185 and .908 . According to the classification proposed by Gil (2011, p.58), most of the items of the test $(n=24)$ would be included in the categories of "very easy" and "easy" -with indices of difficulty greater than .55-. The rest of the items could be considered "normal" $(n=2)$, "difficult" $(n=7)$ and "very difficult" $(n=$ 3 ). It is assumed that the index of difficulty of the items decreases - increasing the difficulty of these- when applying the test to students in lower grades, since, as evidenced in a previous study (Felipe, 2015), the 4th year student's performance in an earlier version of the CLUni Test is superior to the 1st. In the fourth column the indices of discrimination of the items appear. Following, again, the classification proposed by Gil (2011, p. 61), 16 of the items discriminate very well $(n=$

4) or well ( $n=12), 12$ of the items discriminate little and 8 of the items must be improved $(n=6)$ or lack utility to discriminate $(n=2)$. The last column shows the total alpha values of the test associated with the deletion of each item. These values demonstrate that the elimination of some items would mean a minimum increase in the reliability index.

Table 2. Statistics obtained in the analysis of the items of the CLUni Test

\begin{tabular}{|c|c|c|c|c|}
\hline Items & $\begin{array}{c}\text { Index } \\
\text { of difficulty }\end{array}$ & $\begin{array}{l}\text { Standard } \\
\text { Deviation }\end{array}$ & $\begin{array}{c}\text { Index } \\
\text { of discrimination }\end{array}$ & $\begin{array}{c}\text { Cronbach's } \\
\text { Alpha } \\
\text { if the element } \\
\text { has been } \\
\text { annulled }\end{array}$ \\
\hline
\end{tabular}




\begin{tabular}{|c|c|c|c|c|}
\hline 1 &, 323 & ,4713 & ,461 & ,794 \\
\hline 2 & ,462 & ,5024 & ,266 &, 802 \\
\hline 3 & ,908 & ,2917 & ,019 & ,808 \\
\hline 4 & ,615 & ,4903 & ,238 & ,803 \\
\hline 5 & ,708 & ,4584 &, 315 &, 800 \\
\hline 6 & ,754 & ,4341 & ,299 &, 801 \\
\hline 7 &, 323 & ,4713 & ,257 & ,802 \\
\hline 8 & ,692 & ,4651 & ,394 & ,797 \\
\hline 9 & ,615 & 4903 & ,176 & ,806 \\
\hline 10 & ,246 & ,4341 &, 343 & ,799 \\
\hline 11 & ,646 & ,4819 & ,100 & ,809 \\
\hline 12 & ,346 & ,3298 &, 338 &, 800 \\
\hline 13 & ,908 & ,2917 &, 215 & ,803 \\
\hline 14 & ,415 & ,4966 & ,067 &, 810 \\
\hline 15 & ,769 & ,4246 &, 350 & ,799 \\
\hline 16 &, 831 & ,3779 & ,275 & ,801 \\
\hline 17 & ,662 & ,4769 & ,601 & ,788 \\
\hline 18 & ,969 & , 1740 & ,165 &, 805 \\
\hline 19 & ,892 & ,3124 &, 312 &, 801 \\
\hline 20 & ,185 & ,3910 &, 347 & ,799 \\
\hline 21 &, 354 & ,4819 &, 344 & ,799 \\
\hline 22 & ,677 & ,4713 &, 379 & ,797 \\
\hline 23 & ,185 & ,3910 &, 254 & ,802 \\
\hline 24 & ,446 & ,5010 &, 344 & ,799 \\
\hline 25 &, 831 & ,3779 & ,223 & ,803 \\
\hline 26 & ,508 & ,5038 & ,276 & ,802 \\
\hline 27 & ,677 & ,4713 & ,271 & ,802 \\
\hline 28 & ,846 & ,2784 & ,239 &, 803 \\
\hline 29 & ,662 & ,4769 & ,220 & ,804 \\
\hline 30 & ,862 & ,3481 & ,153 & ,805 \\
\hline 31 & ,969 & , 1740 &, 334 & ,802 \\
\hline 32 & ,877 & ,3311 &, 173 & ,804 \\
\hline 33 & ,985 & , 1240 & ,103 & ,806 \\
\hline
\end{tabular}




\begin{tabular}{lllll}
\hline $\mathbf{3 4}$ &, 415 &, 4966 &, 350 &, 799 \\
\hline $\mathbf{3 5}$ &, 554 &, 5010 &, 632 &, 786 \\
\hline $\mathbf{3 6}$ &, 769 &, 4246 &, 496 &, 793 \\
\hline
\end{tabular}

\section{Validity}

The CLUni Test was validated by 6 experts from the areas of Language and Literature Didactics, Hispanic Philology, Linguistics and Communication), who were sent by email the preliminary version of the test (text booklet, question booklet and answer key) and a document - made by following the guidelines and advice of Feliz (2010) - in which they were asked for their collaboration for validation by experts. After a brief introduction describing the test and its recipients, they were presented with a series of tables for a general evaluation of the test and for the specific assessment of each of the texts and the items that refer to them, in terms of its relevance (in which way it assesses reading competence) and adequacy (to the recipients of the test), by means of scales from 1 to 5, in which 1 meant "nothing relevant / adequate" and 5, "very relevant

/ suitable". They also had a space for comments on the test in general and on each text and item, where they could write down what they considered appropriate (reasons for their choice in the scale, proposals for improvement, proposals for change or elimination, errata...). Finally, they were invited to write comments and suggestions.

The experts who participated in the validation process evaluated most of the texts and items that make up the test very positively. Likewise, in their general comments they indicated that they considered the CLUni Test to be a pertinent and valid tool, which stands out for the textual variety, and that has construct validity since its items are in correspondence with the indications that derive from the concept and the capacity that is intended to be measured. They considered, finally, that the test conforms to the intended objectives and that the instrument is prepared (with the exception of slight considerations) for its application and data collection.

However, the experts consulted pointed out certain aspects of improvement, which were considered and a consensus was sought to make the pertinent modifications, such as, for example, the reduction of the time for its realization (which was initially of one hour and a half), due to that the amplitude of the test and the length of some texts can suppose fatigue and an excessive cognitive demand.

Also, the validity of the content was sought through the selection of types of texts and the design of the items according to the assumptions established in the theoretical framework of PISA 2009-2015. 


\section{Evaluation}

The evaluation of the test was carried out according to the total scores obtained by the participants in the study (Table 3). As we can see, the average of the scores was 22.89 out of 36 , which would be equivalent to 6.36 out of 10 . The students who scored better in the test correctly answered 31 of the questions (which would mean a grade of 8.61 out of 10) and students with a lower score answered correctly only 10 items (which would correspond to a grade of 2.78 out of 10 ).

Table 3. Results of the CLUni Test

\begin{tabular}{cccccc}
\hline Mean & $\begin{array}{c}\text { Standard } \\
\text { Deviation }\end{array}$ & Mode & Median & Min. & Max. \\
22,89 & 5,392 & 28,5 & 23 & 10 & 31 \\
\hline
\end{tabular}

\section{Evaluation of the participants}

The students were given a questionnaire to assess aspects of the CLUni Test and answered it at the end of the test, whose findings are presented in this section. Regarding the extension of the test, $29 \%$ of the participants thought it was too long; likewise, $21.9 \%$ stated that their length had affected their performance; those who explained how the length of the test affected them in the performance in the test were referring, in most cases, to that as time went by their concentration and attention decreased, and their fatigue increased. In this regard it should be noted that the average time of completion was 53 minutes. These data -as well as those from the comments of the experts consulted-advise reviewing it with the aim of reducing the time of completion. In another order of things, more than half of the participants (59.4\%) said that the test had been a challenge and, with the exception of one of them, the rest considered it interesting to perform these type of tests. Also the group of participants - except one- expressed their interest in knowing the result of the test.

Also, they were asked to assess the difficulty of the test on a scale of 1 (= No difficulty) to 10 (= much difficulty). From the data obtained, it can be deduced that the group of participants considered that the test presented an average difficulty, although, according to the values of the mode and the median, a considerable number of them stated that the test involved a high difficulty (Table 4). 
Table 4. CLUni Test Evaluation results of perceived difficulty

\begin{tabular}{cccccc}
\hline Mean & $\begin{array}{c}\text { Standard } \\
\text { Deviation }\end{array}$ & Mode & Median & Min. & Max. \\
6,36 & 2,028 & 8 & 7 & 1 & 8 \\
\hline
\end{tabular}

Finally, they were asked to estimate the number of questions they thought they had answered correctly; as can be seen in the results obtained (Table 5), the participants, as a whole, overestimated their performance in this test, which may lead to thinking about the possibility that they also overestimated their level of reading competence.

Table 5. Self-perception of CLUni Test results

\begin{tabular}{cccccc}
\hline Mean & $\begin{array}{c}\text { Standard } \\
\text { Deviation }\end{array}$ & Mode & Median & Min. & Max. \\
28,21 & 5,366 & 30 & 30 & 15 & 36 \\
\hline
\end{tabular}

\section{Conclusion}

This contribution aims to present the process of design and validation of a test of reading competence in the pilot phase aimed at evaluating the reading competence of university students. In light of the results obtained, in the next phase of its development, certain changes in the design of test should be introduced, aimed at eliminating items with a low rate of discrimination and reducing their length to reduce the risk of fatigue affecting performance in the test. Likewise, we are aware that it is necessary to expand the number of participants -as well as their geographical origin as well as university studies- in the research that evaluates the next pilot test in order to increase the representativeness of the sample. However, we understand that the CLUni Test is a contribution to the field of the evaluation of reading competence for a population for which, to date, there is a scarcity of validated instruments.

\section{References}

Arnoux, E. N., Nogueira, S., \& Silvestri, A. (2007). Habilidades metacomprensivas en estudiantes de profesorado: la formulación de preguntas. Folios: Revista de la Facultad de Humanidades, (25), 81-95. 
Calderón, A., \& Quijano, J. (2010). Características de comprensión lectora en estudiantes universitarios. Estudios Socio-Jurídicos, 12(1), 337-364. Retrieved of: http://revistas.urosario.edu.co/index.php/sociojuridicos/article/view/1195

De Brito, N., \& Angeli, A. (2005). Comprensión lectora en universitarios cursantes del primer año en distintas carreras. Paradigma, 26(2), 99-113.

Delgadová, E., \& Gullerová, M. (2015). Comprensión lectora. Un estudio sobre la competencia lectora en el contexto universitario. Lenguajes y Textos, 41, mayo, 4553.

Felipe, A. (2014). Elaboración de una prueba piloto para la competencia lectora de estudiantes universitarios. In Requeijo, P. \& Gaona, C. (coord.), Contenidos innovadores en la Universidad actual (pp. 297-311). Madrid: McGraw-Hill.

Felipe, A., \& Barrios, E. (2015). Prospective teachers' reading competence: percepcions and performance in a reading test. Procedia - Social and Behavioral Sciences, 178, 87-93. Retrieved of: https://www.sciencedirect.com/science/article/pii/S187704281501944

Felipe, A., \& Barrios, E. (2017). Evaluación de la competencia lectora de futuros docentes, Investigaciones Sobre Lectura, 7, 7-21.

Feliz, T. (2010). Validación de un cuestionario por expertos. Retrieved of: https://www.youtube.com/watch?v=C7S5ImJZhvo.

Gil, J. A. (2011). Técnicas e instrumentos para la recogida de información. Madrid: Universidad Nacional de Educación a Distancia.

Irigoyen, J., Jiménez, M., \& Acuña, K. (2008). Análisis de la competencia lectora en estudiantes universitarios. Boletín electrónico de investigación de la Asociación Oaxaqueña de Psicología, 4, 84-96. Retrieved of: http://www.conductitlan.net/52_competencia_lectora.pdf

Martínez, T.; Vidal-Abarca, E., Sellés, P., \& Gilabert, R. (2008). Evaluación de las estrategias y procesos de comprensión: el Test de Procesos de Comprensión. Infancia y Aprendizaje, 31(3), 319-333.

Martínez Solís, M. C. (1997). El desarrollo de estrategias discursivas a nivel universitario. In M. C. Martínez (Comp.), Los procesos de la lectura y la escritura (11-41). Santiago de Cali: Universidad del Valle.

Ministerio de Educación (2010). La lectura en PISA 2009. Marcos y pruebas de evaluación. Madrid. Retrieved of: http://www.mecd.gob.es/dctm/ievaluacion/internacional/lectura-enpisa.pdf?documentId $=0901 \mathrm{e} 72 \mathrm{~b} 8072 \mathrm{f} 8 \mathrm{~d} 9$

Mendelovits, J. y Sainsbury, M. (2013). 'Cómo construir ítems de Lengua', en el seminario Presentación de ítems liberados de PISA, TIMSS y PIRLS. Marco teórico, elaboración y aprovechamiento didáctico, Centro Internacional de Estudios Superiores de Español, Fundación Comillas, Comillas (Cantabria). Disponible en: https://www.youtube.com/watch? $\mathrm{v}=\mathrm{cN} 8 \mathrm{Kgl}-\mathrm{klOY}$ (primera parte) y https://www.youtube.com/watch?v=SN6CXVf_Ei8 (segunda parte). 
Nunnally, J. C. (1978). Psychometric theory (2nd ed.). Nueva York: McGraw-Hill.

OECD (2009). PISA 2009 Assessment Framework - Key competencies in reading, Mathematics and Science. París: PISA, OECD Publishing. Retrieved of: www.oecd.org/dataoecd/11/40/44455820.pdf

OECD (2017). PISA 2015 Assessment and Analytical Framework: Science, Reading, Mathematic, Financial Literacy and Collaborative Problem Solving, revised edition, PISA,

OECD Publishing. Retrieved of: http://dx.doi.org/10.1787/9789264281820-en.

Solé, I. (1992). Estrategias de lectura. Barcelona, España: Graó.

Solé, I. (2012). Competencia lectora y aprendizaje. Revista Iberoamericana de Educación (OEI), 2012, 59, 43-61.

Van Deven A. H., \& Ferry, D. L. (1980). Measuring and Assessing Organizations.

Nueva York: John Wiley \& Sons, Inc.

Velásquez Rivera, M., Cornejo, C., \& Roco, Á. (2008). Evaluación de la competencia lectora en estudiantes de primer año de carreras del área humanista y carreras del área de la salud en tres universidades del Consejo de Rectores. Estudios pedagógicos (Valdivia), 34(1), 123-138.

Weirlich, E. (1976). A text grammar of English. Heidelberg: Quelle and Meyer. 